\title{
Tempo de reação e tempo das provas de 50 e 100 metros rasos do atletismo em federados e não federados
}

\author{
Renata J. Miyamoto ${ }^{1}$ \\ Cássio M. Meira Jr. ${ }^{2}$ \\ https://doi.org/10.5628/rpcd.04.03.42
}

\author{
${ }^{1}$ Universidade do Grande ABC, Departamento de Saúde \\ Santo André/SP \\ ${ }^{2}$ Universidade de São Paulo, Escola de Educação Física e \\ Esporte, Departamento de Pedagogia do Movimento do \\ Corpo Humano, Brasil
}

\begin{abstract}
RESUMO
Com o intuito clarificar algumas questões ainda não totalmente consensuais no que tange ao tempo de reação (TR), especificamente em relação ao nível de habilidade do sujeito e à correlação com o tempo de movimento (TM), o presente trabalho tem por objetivo verificar a ocorrência de relações entre a saída do bloco e o desempenho nas provas de 50 e 100 metros rasos do atletismo em atletas masculinos de diferentes níveis competitivos. Dois grupos foram formados, um composto por 15 atletas não federados e outro por 17 atletas federados. Além da tomada dos tempos totais nas corridas de 50 (TM50) e 100 (TM100) metros rasos, mediu-se o TR de membros inferiores (TRMI) e o TR de membros superiores (TRMS). Os resultados foram organizados pela comparação entre os grupos em cada uma das medidas (teste t Student) e pelo cálculo da matriz de correlação de Pearson nas quatro medidas separadamente para cada grupo. Concluiu-se que: (a) para velocistas de alto nível, quanto menor o TRMI no bloco de saída, maior a chance de sucesso na prova dos 100 metros rasos, e, quanto menor o TM50, melhor é o desempenho nos 100 metros; (b) embora os atletas federados apresentem melhor TM em relação aos não federados, o TR não se configura como uma variável determinante de diferenças de desempenho entre velocistas de níveis diferentes; e (c) TRMS com um movimento simples de mão não se correlaciona com TRMI de saída do bloco de partida.
\end{abstract}

Palavras-chave: tempo de reação, capacidade motora, atletismo, velocidade.

\begin{abstract}
Reaction time and race time on athletics 50 and 100 meters in top level and middle level athletes

In order to clarify some obscure questions related to reaction time (RT), specially to subject's level and the correlation to the movement time $(M T)$, this article aims to verify the relation between the start and the performance of the athletics 50 and 100 meters race in male athletes of different competitive levels. Two groups were formed, one of 15 middle level athletes and other composed of 17 high level athletes. Besides the measurement of the total time of the 50 (MT50) e 100 (MT100) meters race, it was measured $R T$ of the legs (RTL) and RT of the arms (RTA). Results were organised comparing groups in each of the variables ( $t$ Student test) and by means of Pearson correlation matrix on the four variables separately for each group. It can be concluded that: (a) for high level sprinters, the shorter RTL of the starting block, the greater the likelihood of success on the 100 meters race, and, better the time on 50 meters race, better the 100 meters performance; (b) although high level athletes show better MT compared to the middle level athletes, $R T$ is not a determinant variable of performance differences between sprinters of different levels; and (c) RTA with a simple hand movement does not correlate with RTL of the start.
\end{abstract}

Key Words: reaction time, motor ability, athletics, velocity. 


\section{INTRODUÇÃO}

A organização temporal de um movimento em relação à ocorrência de um evento externo pode envolver uma série de atrasos. O intervalo de tempo decorrido entre a apresentação de um estímulo e o início da resposta, denominado tempo de reação (TR), é um dos principais indicadores da limitação neuromotora para processar um estímulo do ambiente externo. Mais especificamente, TR definese como o lapso de tempo entre a apresentação de um estímulo não antecipado, e repentinamente apresentado, e o início da resposta, quando a contração muscular se inicia $(2,9,10,15,16)$.

O TR varia de acordo com alguns fatores, dentre os principais, a modalidade sensorial do estímulo e a complexidade da resposta a ser executada.

Responder a estímulos acústicos, óticos, e táteis implica em TRs diferentes. A despeito de haver controvérsia quanto à quantificação exata, considerando pessoas não treinadas, a amplitude em milissegundos para o TR é de 130 a 170 (acústico sinal sonoro), 200 a 250 (visual - luz), 150 a 160 (tátil simples - toque) e 510 a 530 (tátil complexo - rotação do corpo) $(13,17,18)$. As reações acústicas e óticas diferem umas das outras porque a codificação das ondas luminosas na retina e a condução ao cérebro demoram aproximadamente 30 milissegundos a mais em relação à decodificação das ondas sonoras em impulsos neurais. Há também relação entre o TR e o movimento a ser executado: mantendo constante o estímulo, quanto mais componentes tiver o movimento a ser executado, maior o TR $(6,13)$. Além disso, o TR relaciona-se com os grupos musculares envolvidos na execução do movimento. A hipótese teórica que deriva do processamento de informação é que o TR simples é tanto maior quanto maior a musculatura a ser controlada pelo sistema nervoso. Realmente, segundo os resultados de um experimento clássico (6), executar um movimento com o braço provoca TR de $20 \%$ maior em relação a um movimento com o dedo. Ainda, o processo de crescimento e desenvolvimento causa um decréscimo gradativo do TR: os valores mais baixos, portanto melhores, são alcançados na adolescência e nos primeiros anos da idade adulta, piorando posteriormente na flecha do tempo $(3,7,11)$.
Pode-se subdividir o TR em dois períodos: pré-motor e motor. O primeiro corresponde ao intervalo de tempo entre o disparo do sinal e a primeira mudança no nível de ativação do músculo detectado pela eletromiografia. O segundo refere-se ao intervalo de tempo entre o primeiro sinal eletromiográfico e o início de um movimento perceptível da parte solicitada $(10,14,16)$. Há certa concordância na literatura de que o período pré-motor é um indicador do envolvimento de processos centrais, como recepção e transmissão de informação do ambiente até o próprio músculo, envolvendo o sistema nervoso num processo de tomada de decisões cognitivas e perceptivas durante a preparação do movimento. Esse período é caracterizado pelas seguintes etapas: (a) tempo que o receptor demora a captar o estímulo; (b) tempo que demora a o estímulo chegar desde os receptores da zona cerebral até cada sentido correspondente; (c) tempo de elaboração da resposta (única parte realmente treinável); e (d) tempo que o estímulo demora a percorrer a via aferente até chegar à placa motora (11). O período motor é considerado como o atraso temporal para vencer a inércia do membro, logo após o recebimento do comando para a contração - agem os processos associados com a musculatura em si, isto é, atividades elétricas já detectáveis, mas ainda não traduzidas em movimento. Ressalta-se que apenas a etapa (c) do período pré-motor é sensível ao treinamento, o que caracteriza o TR como uma capacidade motora pouco treinável. De fato, na literatura de comportamento motor, entende-se o TR como uma capacidade motora determinada principalmente por fatores genéticos, portanto pouco afetada pelo processo de prática $(9,10,17)$. O TR tem um importante significado teórico porque envolve mecanismos de processamento, tais como a captação do estímulo pelos órgãos sensoriais, a interpretação do estímulo pelo sistema perceptivo, a tomada de decisão e os responsáveis pela produção do movimento (12). Daí decorre a explicação do porquê não é possível reagir imediatamente a um estímulo; sempre há uma latência relativa ao processamento da informação no sistema nervoso. Já em 1850, Helmholtz concluiu, depois da realização de um experimento de estimulação da parte inferior do pé, que o impulso neural viaja à velocidade de 50 a $100 \mathrm{~m} / \mathrm{s}$ (12). Daí depreende, portanto, que o tempo de condução nervosa periférica por si só explicaria uma parte do TR. A considerar 
que qualquer TR total sempre compreende mais que 100 milissegundos, a outra parte do TR (e maior) seria devida ao processamento no sistema nervoso central. E qualquer fator que prolongue um ou mais desses mecanismos será responsável também pelo aumento do TR.

Existem três tipos de TR: simples (somente uma resposta a somente um estímulo - por exemplo, a saída de provas de atletismo e natação); de escolha (há mais de um estímulo e cada estímulo exige uma resposta específica ao estímulo particular, isto é, há necessidade de compatibilizar a resposta ao estímulo - por exemplo, a recepção a diferentes tipos de saque ou a defesa de golpes); e de discriminação (há mais de um estímulo, porém somente uma resposta - por exemplo, a largada de corridas de automobilismo). Em provas curtas do atletismo, uma vitória pode ser definida em décimos ou centésimos de segundos. A saída do bloco de partida estabelece o início da prova, e um dos componentes essenciais dessa fase inicial é o TR. Logo, minimizar o TR em situações como essa implica na obtenção de uma vantagem significativa. Entretanto, como Carl Lewis na prova dos 100 metros e Tim Harden na prova dos 60 metros, há corredores que, embora reajam mais lentamente ao estímulo sonoro do tiro, são capazes de recuperar essa deficiência no decorrer da prova e vencê-la (Tim Harden, quando obteve o título do campeonato mundial indoor, em 2001, apresentou o pior TR dentre todos os finalistas: 135 milissegundos). Existem também outros casos, como os de Tim Montgomery, em que a reação ao tiro foi tão rápida (104 milissegundos) na final do Grand Prix da Associação Internacional das Federações de Atletismo [International Association of Athletics Federations - IAAF (8)], em 2002, que o permitiu tomar a dianteira em relação aos outros corredores, fato que em nível internacional pode ser decisivo no alcance da vitória - a propósito, com o tempo de 9,780 segundos, ele é o detentor do recorde mundial dos 100 metros rasos. Portanto, estabelece-se um paradoxo: se por um lado um corredor que reaja atrasado ao disparo ainda pode ter chances de ganhar a prova, por outro, um corredor que reaja rapidamente ao tiro pode perdê-la.

Vale ressaltar que o TR inclui não o movimento em si, mas somente o tempo antes de o movimento começar. O tempo total da prova é composto, além do TR, pelo tempo de movimento (TM - intervalo de tempo transcorrido desde a primeira contração muscular até o cruzamento da linha de chegada). Em poucas palavras, o TM se inicia quando o TR termina. Obviamente, o TM também é de vital importância para o bom desempenho da prova, haja vista que ele se constitui em $99 \%$ do tempo da prova masculina - o $1 \%$ restante é atribuído ao TR. Portanto, capacidades físicas altamente sensíveis ao treinamento - por exemplo, velocidade e força - determinam de forma decisiva o resultado final em provas de velocidade. Na literatura, o TR e o TM são entendidos como medidas independentes, o que teoricamente pressupõe baixa correlação entre elas $(4,5)$. Relação bastante questionada é a do TR com o nível de habilidade do executante. É sabido que a capacidade de antecipação adquirida por meio da experiência de indivíduos habilidosos faz com esses tenham TR menor em relação a indivíduos em estágios iniciais de prática, independentemente da complexidade da tarefa $(11,17)$. Assumindo que o atleta é um processador de informação (12), iniciantes requerem um tempo de processamento de informações maior em comparação a atletas treinados. Os atletas mais experientes apresentam maior velocidade do processamento da informação em todas as fases do tempo de reação, desde a recepção do estímulo, de condução até o sistema nervoso central, do processamento dos mesmos e da mobilização dos músculos efetores. Entretanto, com base nos resultados de recentes provas de velocidade no atletismo (8), pode-se dizer que não há padrão de correlação entre o TR de atletas de diferentes níveis.

Com o propósito de esclarecer algumas questões ainda não totalmente consensuais no que tange ao TR, especificamente em relação ao nível de habilidade do sujeito e à correlação com o TM, o presente trabalho tem por objetivo verificar a ocorrência de relações entre a saída do bloco e o desempenho nas provas de 50 e 100 metros rasos do atletismo em atletas de diferentes níveis.

\section{METODOLOGIA}

\section{Sujeitos}

Fizeram parte da pesquisa 32 velocistas da modalidade esportiva atletismo, todos do sexo masculino (média de idade de 21,75 anos; desvio padrão de 5,52 anos). Um grupo foi composto de 17 atletas de 
alto nível, federados e participantes de competições em níveis estadual, brasileiro e internacional (treinavam em média 6 vezes por semana e 4 horas por dia). O outro grupo foi composto por 15 atletas, não federados e participantes de competições a nível estadual (treinavam em média 2 vezes por semana e 3 horas por dia).

\section{Material e métodos}

O TR e o TM foram mensurados numa pista oficial de atletismo, com a utilização de dois blocos de partida e um aparelho de medição do tempo de reação

(Reaction/Movement Timer - Lafayette Instruments). O aparelho é constituído de duas chaves de resposta (A e B) e um emissor de estímulo, conectados a um painel de controle. Além disso, os seguintes materiais foram utilizados: dois suportes de ferro, uma fita K-7, duas chaves micro switch (linha miniatura), 100 metros de fio tipo jumper cor branca e fitas adesivas. Cada atleta percorreu a distância de 50 metros rasos, com velocidade máxima, saindo de um bloco de partida, em posição agachada, logo após um sinal sonoro. A chave "A" estava posicionada no bloco de partida, juntamente onde o atleta a pressionava e posicionava o pé traseiro. Logo depois de emitido o estímulo sonoro, acionavam-se o primeiro e o segundo cronômetro do aparelho. O tempo de reação de membros inferiores (TRMI) foi medido pelo primeiro cronômetro, desde o momento do estímulo até a retirada do pé do bloco. O cronômetro fez a medição a partir do estímulo sonoro até a descompressão da chave "A". Foi solicitado um número total de cinco saídas de bloco. Somente na última tentativa o atleta percorreu os 50 metros completos. Na linha de chegada dos 50 metros, o atleta ultrapassava uma linha, momento em que pressionava a chave "B", parando assim o segundo cronômetro. O segundo cronômetro mediu o tempo do atleta na corrida de 50 metros, ou seja, o seu tempo de movimento (TM50). Como medida complementar, solicitou-se a medida do tempo de reação dos membros superiores (TRMS). Para esta medição, foi utilizado o mesmo aparelho de medida de TRMI. Pediu-se para o atleta pressionar sua mão inteira sobre o aparelho, com os dedos voltados para baixo. Depois de dado o sinal de "pronto", o aparelho disparava o sinal sonoro, após o qual, o atleta deveria retirar sua mão dominante o mais rápido possível. As cinco tentativas foram executadas sempre com a mesma mão, logo após a medida de TRMI.

Para obtenção dos tempos dos 100 metros rasos, perguntou-se ao atleta sua melhor marca obtida durante todo seu período de prática da modalidade.

\section{RESULTADOS}

Nas Tabelas 1 e 2 são apresentados os resultados de cada atleta de ambos os grupos nas variáveis TRMI, TRMS, TM50, TM100. É importante ressaltar que, devido a uma limitação técnica do aparelho de medição (a reação foi detectada apenas após a descompressão da chave e não pela detecção imediata de mudança de pressão), os valores de TR da presente amostra são maiores em comparação com valores oficiais de provas de atletismo.

Tabela 1 - Médias e desvios-padrão nas variáveis TRMI, TRMS, TM50, TM100 para atletas federados.

\begin{tabular}{c|c|c|c|c} 
ATLETA & TRMI & TM50 & TM100 & TRMS \\
\hline $\mathbf{1}$ & $0,320 \pm 0,003$ & 6,235 & 10,600 & $0,194 \pm 0,006$ \\
$\mathbf{2}$ & $0,352 \pm 0,015$ & 6,722 & 10,657 & $0,214 \pm 0,010$ \\
$\mathbf{3}$ & $0,316 \pm 0,011$ & 6,355 & 10,657 & $0,196 \pm 0,010$ \\
$\mathbf{4}$ & $0,344 \pm 0,018$ & 6,348 & 10,657 & $0,181 \pm 0,014$ \\
$\mathbf{5}$ & $0,301 \pm 0,010$ & 6,280 & 10,490 & $0,228 \pm 0,004$ \\
$\mathbf{6}$ & $0,351 \pm 0,001$ & 6,377 & 10,657 & $0,190 \pm 0,007$ \\
$\mathbf{7}$ & $0,360 \pm 0,032$ & 6,537 & 10,910 & $0,213 \pm 0,010$ \\
$\mathbf{8}$ & $0,372 \pm 0,007$ & 6,645 & 10,657 & $0,211 \pm 0,007$ \\
$\mathbf{9}$ & $0,330 \pm 0,006$ & 6,536 & 10,270 & $0,209 \pm 0,007$ \\
$\mathbf{1 0}$ & $0,324 \pm 0,001$ & 6,092 & 10,490 & $0,178 \pm 0,007$ \\
$\mathbf{1 1}$ & $0,333 \pm 0,007$ & 6,654 & 10,850 & $0,189 \pm 0,003$ \\
$\mathbf{1 2}$ & $0,371 \pm 0,007$ & 6,691 & 10,950 & $0,154 \pm 0,047$ \\
$\mathbf{1 3}$ & $0,275 \pm 0,051$ & 6,285 & 10,190 & $0,211 \pm 0,009$ \\
$\mathbf{1 4}$ & $0,327 \pm 0,014$ & 6,311 & 10,740 & $0,185 \pm 0,010$ \\
$\mathbf{1 5}$ & $0,302 \pm 0,058$ & 6,737 & 10,900 & $0,213 \pm 0,007$ \\
$\mathbf{1 6}$ & $0,355 \pm 0,017$ & 6,519 & 10,657 & $0,193 \pm 0,005$ \\
$\mathbf{1 7}$ & $0,406 \pm 0,050$ & 6,525 & 10,840 & $0,259 \pm 0,009$
\end{tabular}


Tabela 2 - Médias e desvios-padrão nas variáveis TRMI, TRMS, TM50, TM100 para atletas não federados.
Tabela 3 - Valores de média, desvio padrão, t value e p de TRMI, TRMS, TM50 e TM100 para os grupos de atletas federados e não federados.

\begin{tabular}{c|c|c|c|c} 
ATLETA & TRMI & TM50 & TM100 & TRMS \\
\hline $\mathbf{1}$ & $0,326 \pm 0,008$ & 6,371 & 11,500 & $0,188 \pm 0,011$ \\
$\mathbf{2}$ & $0,325 \pm 0,032$ & 6,858 & 11,300 & $0,203 \pm 0,016$ \\
$\mathbf{3}$ & $0,273 \pm 0,055$ & 6,569 & 11,300 & $0,189 \pm 0,011$ \\
$\mathbf{4}$ & $0,244 \pm 0,010$ & 6,662 & 11,800 & $0,205 \pm 0,008$ \\
$\mathbf{5}$ & $0,343 \pm 0,064$ & 6,825 & 11,370 & $0,224 \pm 0,012$ \\
$\mathbf{6}$ & $0,383 \pm 0,007$ & 6,809 & 11,100 & $0,252 \pm 0,015$ \\
$\mathbf{7}$ & $0,317 \pm 0,007$ & 6,934 & 11,800 & $0,180 \pm 0,019$ \\
$\mathbf{8}$ & $0,345 \pm 0,012$ & 6,544 & 11,400 & $0,225 \pm 0,007$ \\
$\mathbf{9}$ & $0,351 \pm 0,009$ & 6,636 & 11,500 & $0,194 \pm 0,004$ \\
$\mathbf{1 0}$ & $0,376 \pm 0,005$ & 7,059 & 12,120 & $0,209 \pm 0,008$ \\
$\mathbf{1 1}$ & $0,310 \pm 0,010$ & 7,122 & 12,000 & $0,187 \pm 0,013$ \\
$\mathbf{1 2}$ & $0,334 \pm 0,013$ & 6,630 & 11,450 & $0,195 \pm 0,011$ \\
$\mathbf{1 3}$ & $0,346 \pm 0,009$ & 6,929 & 12,000 & $0,197 \pm 0,010$ \\
$\mathbf{1 4}$ & $0,364 \pm 0,005$ & 6,658 & 12,100 & $0,177 \pm 0,002$ \\
$\mathbf{1 5}$ & $0,345 \pm 0,019$ & 6,785 & 12,100 & $0,190 \pm 0,009$
\end{tabular}

Uma vez que não foi possível detectar diferenças entre os grupos por meio da estatística descritiva (Tabela 3) e considerando o reduzido número de componentes de cada grupo, os dados foram submetidos ao teste Shapiro-Wilk's W, o qual detectou normalidade da distribuição em todas as variáveis ( $p>0,05)$. Em vista disso, foi possível utilizar o teste paramétrico $t$ Student para amostras independentes. Não foram verificadas diferenças estatisticamente significativas entre os atletas federados e os não federados nas variáveis TRMI $(t$-value $=0,45 ; \mathrm{p}=0,65)$ e TRMS ( $t$-value $=-0,00068 ; \mathrm{p}=0,99)$. Em contrapartida, houve diferença significativa na variável TM50 $(t$-value $=4,24 ; \mathrm{p}<0,01)$ : os atletas federados correram os 50 metros rasos mais rápido em comparação com os atletas não federados. Diferença significativa também foi detectada no TM100 ( $t$-value $=9,96$; $\mathrm{p}<0,01)$. Novamente, o grupo de federados foi mais rápido nos 100 metros rasos em comparação ao grupo de não federados.

\begin{tabular}{l|c|c|c|c} 
& $\begin{array}{c}\text { Atletas } \\
\text { federados }\end{array}$ & $\begin{array}{c}\text { Atletas não } \\
\text { federados }\end{array}$ & t value & $p$ \\
\hline TRMI & $0,337 \pm 0,031$ & $0,332 \pm 0,036$ & 0,450 & 0,650 \\
TRMS & $0,201 \pm 0,023$ & $0,201 \pm 0,019$ & 0,000 & 0,990 \\
TM50 & $6,462 \pm 0,192$ & $6,759 \pm 0,204$ & 4,240 & $<0,010^{*}$ \\
TM100 & $10,657 \pm 0,211$ & $11,656 \pm 0,347$ & 9,960 & $<0,010^{*}$ \\
\multicolumn{4}{c}{ *significância estatística }
\end{tabular}

As quatro variáveis dependentes (TRMI, TRMS, TM50 e TM100) foram correlacionadas separadamente para cada grupo por meio do índice de correlação de Pearson (Tabelas 4 e 5).

Tabela 4 - Correlações de Pearson das variáveis TRMI, TRMS, TM50 E TM100 para atletas federados.

\begin{tabular}{l|c|c|c|c} 
& TRMI & TRMS & TM50 & TM100 \\
\hline TRMI & $\cdot$ & 0,12 & 0,44 & $0,55^{*}$ \\
TRMS & & $\cdot$ & 0,13 & $-0,12$ \\
TM50 & & & $\cdot$ & $0,54^{*}$ \\
TM 100 & & & & $\cdot$ \\
& & &
\end{tabular}

Tabela 5 - Correlações de Pearson das variáveis TRMI, TRMS, TM50 \& TM100 para atletas não federados.

\begin{tabular}{l|c|c|c|c} 
& TRMI & TRMS & TM50 & TM100 \\
\hline TRMI & & 0,34 & 0,21 & 0,05 \\
TRMS & & $\cdot$ & $-0,09$ & $-0,56 *$ \\
TM50 & & & $\cdot$ & 0,45 \\
TM100 & & & & - \\
& & & \\
& & & \\
& & & \\
& & &
\end{tabular}

Para atletas federados foram detectadas correlações significativas e positivas, porém fracas, entre TRMI e TM100 e entre TM50 e TM100. No que tange ao grupo de não federados, foi identificada apenas uma correlação significativa e negativa, porém fraca, entre TRMS e TM100. 


\section{DISCUSSÃO}

Com resultados de correlação moderada ou até mesmo inexistente entre TR e TM, pode-se dizer que as duas habilidades correlacionadas (a saída do bloco e a corrida) são, de certa forma, independentes uma da outra $(4,5)$. De acordo com a literatura, as duas habilidades - saída do bloco e corrida - são sustentadas por capacidades diferentes; a saída é determinada predominantemente pela capacidade motora "tempo de reação", que tem como característica ser pouco modificável; por sua vez, o tempo de movimento (corrida) é determinado predominantemente por capacidades físicas bastante sensíveis ao treinamento - sobretudo, força e velocidade.

É importante destacar que não houve correlação entre TRMI e TRMS para ambos os grupos, que mesmo subsidiados pela mesma capacidade motora, tempo de reação, não apresentaram correlação significativa. Esse resultado não corrobora a hipótese de que o TR é tanto maior quanto maior a musculatura envolvida na execução do movimento (6). A propósito, essa constatação apenas é válida quando a comparação considera os membros superiores. No presente estudo, a comparação deu-se entre um movimento com a mão dominante (membro superior) e um movimento específico de saída do bloco de partida (membro inferior). Ainda, é possível fornecer uma explicação alternativa à ausência de correlação entre TRMI e TRMS com base no fato de que os componentes de ambos os grupos treinavam sistematicamente. Se os atletas não federados não treinassem sistematicamente, haveria maior probabilidade de ocorrer diferença entre os grupos, sobretudo no TRMI, mais sujeito à influência do treinamento (17). Outro objeto de interesse do presente estudo são as relações entre TR, TM e nível de habilidade do executante. O grupo de federados percorreu em média, as distâncias de 50 e 100 metros em tempos menores que o grupo de não federados, ou seja, o grupo de federados foi significativamente mais rápido em relação ao grupo de não federados. Nesta variável, especificamente, era de se esperar esse resultado porque os atletas federados treinam com mais freqüência em comparação aos não federados.

Teoricamente, como nenhum indivíduo da amostra se encontrava em estágios iniciais de prática na modalidade, a diferença entre os grupos não deveria ser detectada para o TR $(11,17)$, e, de fato, os resul- tados referentes ao TRMI e ao TRMS sustentaram essa hipótese. Pode-se especular que, em todos os sujeitos da amostra, devido à pouca sensibilidade ao treinamento, o pequeno limite de melhora no TR já tenha sido alcançado em virtude da prática sistemática ao qual eles se submetem. Ressalta-se que o treinamento tem uma importância vital, não para melhorar significativamente o TR, mas para mantêlo em seus melhores níveis $(11,17)$. A prática faz com que o executante saiba lidar com as dicas do ambiente, isto é, a discriminação do que é e do que não é relevante é aperfeiçoada $(1,19)$.

Diferentemente do TR de escolha e do TR de discriminação, os quais são sensivelmente afetados pela quantidade de prática do executante $(10,16,18)$, o processamento da informação no TR simples não envolve o mecanismo de tomada de decisão, fator preponderante de diferenças significativas entre iniciantes e habilidosos $(10,12,14,19)$. No TR simples, o executante não toma decisão alguma, apenas espera o estímulo e inicia o movimento.

\section{CONCLUSÕES}

O nível de habilidade do velocista de atletismo não se correlaciona com o TR, mas, como esperado, apenas com o tempo da prova. Especificamente para atletas de alto nível, o TR na saída do bloco está associado ao tempo de movimento de 100 metros rasos. No entanto, a correlação entre as duas variáveis é bastante tênue.

Embora os atletas federados apresentem melhor TM em relação aos não federados, o TR não se configura como uma variável determinante de diferenças de desempenho entre velocistas de níveis diferentes. Além disso, possuir bom TR com um movimento simples com a mão não implica em reagir mais rápido ao estímulo na saída da prova.

\section{CORRESPONDÊNCIA}

Cássio M. Meira Jr.

Universidade de São Paulo

Escola de Educação Física e Esporte

Departamento de Pedagogia do Movimento do Corpo Humano

Av. Prof. Mello Moraes 65 - Cid. Universitária São Paulo/SP - 05508-900 - BRASIL cmj@usp.br 


\section{REFERÊNCIAS BIBLIOGRÁFICAS}

1. Abernethy B (1990). Anticipation in squash: differences in advance cue utilization between expert and novice players. Journal of Sports Sciences 8: 17-34.

2. Fleishman EA (1972). On the relation between abilities, learning, and human performance. American Psychologist 27: 1017-1032.

3. Gallahue, DL, Ozmun JC (2001). Compreendendo o desenvolvimento motor: bebês, crianças, adolescentes e adultos. São Paulo: Phorte.

4. Henry FM (1960). Influence of motor and sensory sets on reaction latency and speed of discrete movements. Research Quarterly 31: 459-468.

5. Henry FM (1961). Stimulus complexity, movement complexity, age, and sex in relation to reaction latency and speed in limb movements. Research Quarterly 32: 353-366.

6. Henry FM, Rogers DE (1960). Increased response latency for complicated movements and the "memory drum" theory of neuromotor reaction. Research Quarterly 31: 448-458.

7. Hodgkins J (1962). Influence of age on the speed of reaction time and movement in females. Journal of Gerontology 17: 385-389.

8. International Association of Athletics Federations - IAAF (2003). The sport of athletics. [on line]. [2003, 19 de novembro]. Disponível: www.iaaf.org

9. Magill RA (1984). Aprendizagem motora: conceitos e aplicações. São Paulo: Edgard Blücher.

10. Magill RA (2000). Aprendizagem motora: conceitose aplicações. São Paulo: Edgard Blücher.

11. Manso JMG, Acero RM, Valdivielso MN, Caballero JAR (1998). La velocidad: la mejora del rendimiento en los deportes de velocidad. Madrid: Gymnos.

12. Marteniuk RG (1976). Information processing in motor skill. New York: Holt, Rinehart \& Winston.

13. Schmidt RA (1982). Motor control and learning: a behavioral emphasis. Champaign: Human Kinetics.

14. Schmidt RA (1988). Motor control and learning: a behavioral emphasis. $2^{\text {nd }}$. Ed. Champaign: Human Kinetics.

15. Schmidt RA (1993). Aprendizagem e performance motora: dos princípios à prática. São Paulo, Movimento.

16. Schmidt RA, Wrisberg CA (2001). Aprendizagem e performance motora: uma abordagem da aprendizagem baseada no problema. Porto Alegre: Artmed.

17. Weineck J (1999). Treinamento ideal. São Paulo: Manole.

18. Welford AT (1980). Reaction times. London: Academic Press.

19. Williams AM, Davids K, Williams JG (1999). Visual perception $\mathcal{E}$ action in sport. London: E \& FN Spon - Routledge. 